\title{
CISTICERCOSIS DISEMINADA: REPORTE DE UN CASO EN PERÚ
}

\author{
Julio Maquera-Afaray',2,a, Edwin Capaquira ${ }^{3, b}$, Li Conde ${ }^{1, c}$
}

\begin{abstract}
RESUMEN
La cisticercosis es una enfermedad desatendida y endémica en el Perú que afecta comúnmente al sistema nervioso central (SNC), causando la neurocisticercosis (NCC). Sin embargo, son pocos los reportes de cisticercosis diseminada (CCD) en el mundo. Se reporta el caso de un paciente varón de 82 años, natural del departamento de Junín, que presenta pérdida brusca del nivel de conciencia asociada a convulsiones tónico-clónicas generalizadas. La tomografía axial computarizada y la resonancia magnética nuclear cerebral mostraron múltiples lesiones de aspecto quístico con presencia de escólex en su interior y compatibles con NCC masiva; la resonancia magnética nuclear torácica, abdominal y pélvica permitieron evidenciar la diseminación multiorgánica de cisticercos. Aunque es poca la casuística y experiencia terapéutica en los casos de CCD, el paciente recibió tratamiento antiparasitario con albendazol y corticoides, logrando una evolución clínica favorable y sin complicaciones durante la hospitalización.
\end{abstract}

Palabras clave: Cisticercosis; Taenia solium; Perú, Neurocisticercosis (fuente: DeCS BIREME).

\section{DISSEMINATED CYSTICERCOSIS: REPORT OF A CASE IN PERU}

\begin{abstract}
Cysticercosis is a neglected and endemic disease in Peru that commonly affects the central nervous system (CNS), causing neurocysticercosis (NCC). However, only a few reports of disseminated cysticercosis (DCC) exist in the world. In this article we present a case report of a male, 82 year old patient from the department of Junin (Peru). He presented a sudden loss of consciousness associated with generalized tonic-clonic seizures. CT scan and brain MRI showed multiple cystic lesions with scolex presence inside and compatible with massive NCC. Thoracic, abdominal and pelvic MRI showed multi-organ dissemination of cysticerci. Although there is little therapeutic experience in cases of DCC, the patient received deworming treatment with albendazole and corticoids, in this way a favorable clinical outcome was achieved without complications during hospitalization.
\end{abstract}

Key words: Cysticercosis; Taenia solium; Peru, neurocysticercosis (source: MeSH NLM).

\section{INTRODUCCIÓN}

La cisticercosis es una zoonosis parasitaria desatendida asociada con la pobreza, afecta a millones de personas en países en desarrollo, y es endémica en Latinoamérica, Asia y África, con una seroprevalencia estimada entre el 10 a $20 \%$. En Perú, las regiones comprometidas se encuentran en toda la sierra, costa norte y selva alta ${ }^{(1,2)}$.

El desarrollo de morbilidad asociada al cisticerco de Taenia solium depende del órgano que afecte, el sistema nervioso central (SNC) es el más infectado causando la neurocisticercosis (NCC) que es la parasitosis más frecuente del SNC y una causa importante de epilepsia adquirida en el mundo. En Latinoamérica, la NCC es más frecuente, a diferencia de la cisticercosis subcutánea que es más común en Asia. Asimismo, se ha estimado en Latinoamérica que alrededor de 400000 están afectados de forma sintomática. No obstante, poco más de 60 casos de cisticercosis diseminada (CCD) han sido reportados en el mundo ${ }^{(3-6)}$.

Reportamos un caso de CCD con extenso compromiso cerebral, ocular, cardiaco, pulmonar, hepático, pancreático, muscular y subcutáneo, en un paciente adulto mayor de un área endémica del Perú.

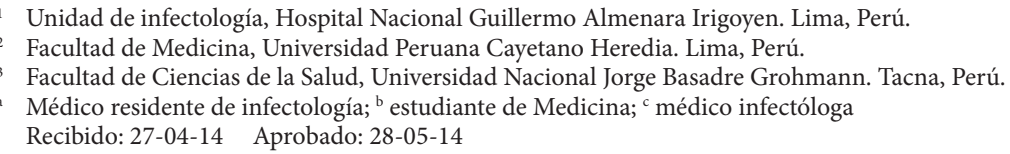


(A)

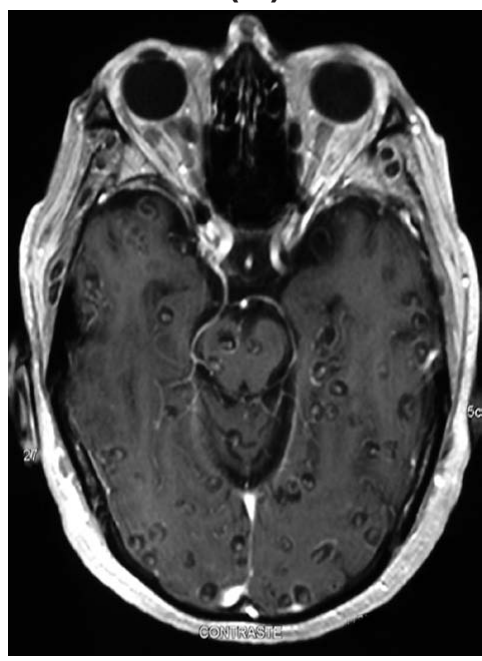

(B)

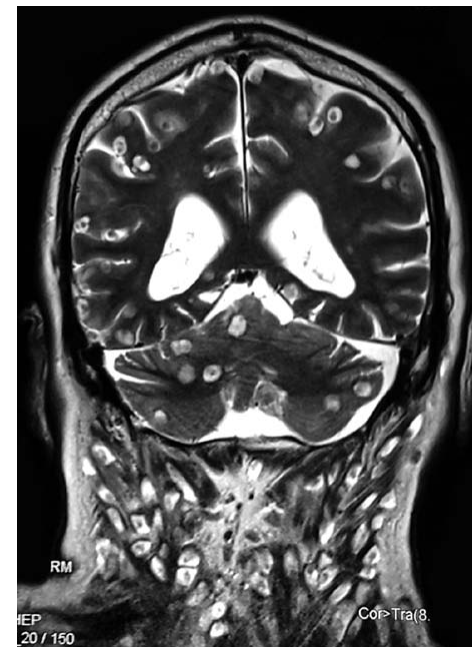

(C)

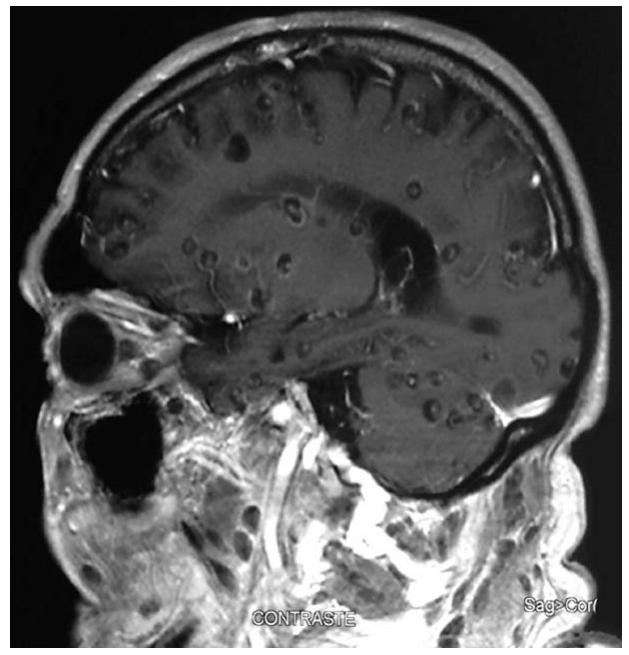

Figura 1. Resonancia magnética nuclear cerebral (A) corte axial, muestra múltiples quistes hipointensos con evidencia de imágenes puntiformes en su interior (escólex) en T1 e hiperintensas en T2, (B) corte coronal comprometiendo hemisferios cerebrales, tronco encefálico y cerebelo, (C) adicionalmente, se observan cisticercos intraorbitarios y en los compartimientos musculares y subcutáneos del cuello y macizo facial

\section{REPORTE DE CASO}

Paciente varón de 82 años de edad, procedente de la ciudad de Huancayo en el departamento de Junín, con historia de deterioro cognitivo progresivo desde hace aproximadamente 5 años. Ingresa al Servicio de Emergencia del Hospital Nacional Guillermo Almenara Irigoyen (categoría III-1), por presentar, de forma brusca, pérdida del nivel de conciencia asociada con convulsiones tónico-clónicas y posterior hemiparesia derecha. Al examen físico se encontraba afebril y con resto de funciones vitales estables; en la piel se hallaron múltiples nódulos subcutáneos palpables en tronco y extremidades, a la evaluación neurológica se encontró alterado el estado de conciencia, somnoliento con desorientación parcial en tiempo y espacio, y disminución de la fuerza muscular en hemicuerpo derecho. Se indican medidas generales y manejo anticonvulsivante.

Los estudios de laboratorio mostraron un hemograma con leucocitos en $9350 \mathrm{cel} / \mathrm{mL}$, con eosinófilos $22 \%$; hemoglobina $12,3 \mathrm{~g} / \mathrm{dL}$ y recuento de plaquetas en 285 000; creatinina en $0,7 \mathrm{mg} / \mathrm{dL}$, glucosa $88 \mathrm{mg} / \mathrm{dL}$, urea $20 \mathrm{mg} / \mathrm{dL}$. El perfil hepático, de coagulación y electrolitos séricos, no presentaron alteraciones. Además, se realizó la prueba de Western Blot para cisticercosis, con resultado positivo. Los estudios imagenológicos, tanto la tomografía axial computarizada (TAC) y la resonancia magnética nuclear (RMN) cerebral mostraron múltiples lesiones de aspecto quístico supra e infratentorial en hemisferios cerebrales, tallo encefálico y cerebelo, con evidencia de escólex en su interior, compatibles con NCC masiva; a nivel extracraneal se observó presencia de lesiones en órbitas, músculos y partes blandas de la cabeza y cuello (Figura 1). Adicionalmente, se realizó RMN de tórax, abdomen y pelvis, que revelaron diseminación masiva de cisticercos con compromiso pulmonar, cardiaco, hepático, pancreático, muscular y subcutáneo (Figuras 2 y 3 ). Se realizó biopsia de los nódulos subcutáneos del antebrazo derecho con resultado anatomopatológico confirmatorio para cisticercosis subcutánea.
(A)

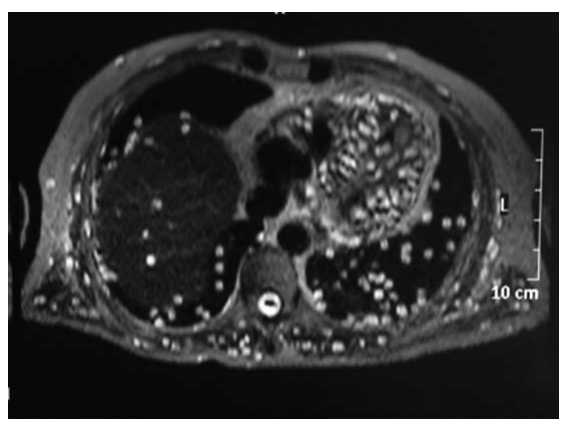

(B)

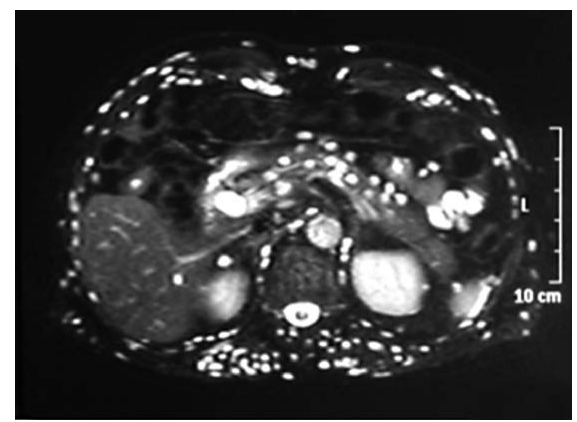

Figura 2. Resonancia magnética nuclear de tórax y abdomen, en las imágenes transversales con técnica de supresión grasa se observa, (A) compromiso pulmonar, cardiaco, hepático, (B) pancreático, muscular y partes blandas 
(A)

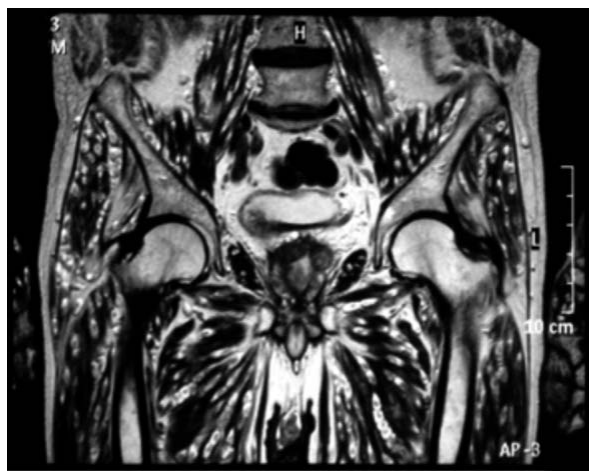

(B)

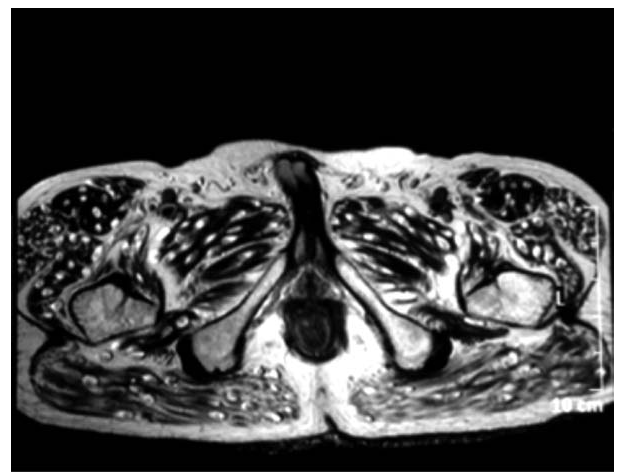

Figura 3. Resonancia magnética nuclear de pelvis $(A)$ corte coronal y (B) transversal en T2 se aprecia infestación masiva de cisticercos a nivel muscular y subdérmico
Tras la evaluación del caso, se decide el inicio de tratamiento antiparasitario con albendazol (15 mg/kg/día, por 2 semanas), previo uso de corticoides (prednisona $60 \mathrm{mg} / \mathrm{día}$, con reducción gradual de dosis) y fenitoína como anticonvulsivante. Durante su hospitalización el paciente completa el tratamiento antiparasitario con evolución clínica favorable y sin complicaciones, llegando a recuperar su estado cognitivo basal (despierto, obedece órdenes simples y moviliza extremidades); no presentó nuevo episodio convulsivo, y fue dado de alta con continuación de terapia anticonvulsivante. Sin embargo, el paciente fallece meses después producto de una neumonía.

\section{DISCUSIÓN}

La CCD es una manifestación rara de la enfermedad incluso en zonas endémicas, con pocos casos documentados en India, Asia, África y Latinoamérica ${ }^{(7-10)}$. En Perú existen algunos reportes de CCD (11-13); sin embargo, este es el primer caso documentado de CCD con extenso compromiso multiorgánico.

Aunque la mayoría de casos de cisticercosis se encuentran en los grupos etarios de 11 a 60 años, con predominio entre los 21 y 40 años; la inmunosenescencia aumenta la susceptibilidad de las personas a desarrollar neoplasias e infecciones. Cavellani et al., demostró que la cisticercosis no es infrecuente en personas de edad avanzada ( $\geq 60$ años) en áreas endémicas y, probablemente estén infectándose continuamente a medida que envejecen. Asimismo, aunque los trastornos de encefalopatía y demencia son más frecuentes en esta población, la alteración cognitiva es también una forma de manifestación clínica de la NCC, y de importancia en zonas endémicas ${ }^{(14,15)}$. Es probable que el deterioro progresivo de las funciones cerebrales superiores del paciente sea debido al compromiso cerebral de la cisticercosis.
Las manifestaciones clínicas de la cisticercosis dependen del órgano que afecte; la NCC y la cisticercosis oftálmica están asociadas con mayor morbilidad, a diferencia de las cisticercosis extraneurales que usualmente son asintomáticas. En la NCC eso se debe fundamentalmente a la interacción entre las características del parásito dentro del SNC y la respuesta inmune del huésped, donde las manifestaciones pueden ser variables, pero las convulsiones son la forma de presentación más común, pues ocurren hasta en $70 \%$ de los casos y, aunque en los adultos mayores existen otras etiologías que comprometen con mayor frecuencia el SNC, la NCC en zonas endémicas se debe descartar en un paciente que convulsiona. Por otro lado, la afección oftálmica provoca fundamentalmente defectos progresivos en la agudeza visual, y la cisticercosis subcutánea y muscular suelen ser asintomáticas ${ }^{(1-3,16)}$. No obstante, son raros los casos descritos con compromiso pulmonar, cardiaco, hepático y pancreático ${ }^{(5,10,17)}$.

A diferencia de otras enfermedades infecciosas desatendidas, la cisticercosis requiere de técnicas diagnósticas avanzadas (TAC y/o RMN) en poblaciones de alto riesgo que también tienen limitaciones económicas ${ }^{(18)}$, por lo cual, resulta complicado, y quizá poco necesario, evaluar la posible diseminación de la enfermedad a otros órganos en un paciente con NCC sin manifestaciones extraneurales, y sea una explicación a la escasez de reportes de CCD. Contrariamente, al diagnosticarse cisticercosis extraneural debería evaluarse el SNC con TAC y/o RMN, al ser la NCC la forma de presentación más común y sintomática de la enfermedad.

Como se mencionó, el diagnóstico de la NCC es típicamente realizado por imágenes, con TAC y/o RMN cerebral; sin embargo, las TAC de pobre resolución podrían, por el contrario, dificultar el diagnóstico. Además, según el tipo de lesión se puede diferenciar con otras enfermedades. La edad del paciente con compromiso neurológico focal y TAC cerebral con múltiples lesiones 
intraparenquimales orientó entre los diagnósticos iniciales una metástasis cerebral. Los estudios serológicos son de utilidad para confirmar el diagnóstico; actualmente, la técnica de inmunoelectrotransferencia (EITB), llamada también Western Blot, es criterio diagnóstico por su alta sensibilidad y especificidad en 98 y $100 \%$, respectivamente. En los casos de cisticercosis subcutánea, el estudio de biopsia confirma el diagnóstico ${ }^{(3,16)}$.

A diferencia de la NCC, la cisticercosis fuera del SNC suele no requerir de tratamiento antiparasitario, no obstante, es poca la casuística y experiencia terapéutica en los casos de CCD, más aun en casos con extenso compromiso de órganos, donde es controversial la decisión terapéutica del uso de antiparasitarios. Cabe mencionar, que en la NCC el manejo tanto sintomático como antiparasitario debe ser individualizado, evaluando las características de las lesiones quísticas (número, tamaño, localización y estado) (16). Además, al enfocar el manejo de los casos de NCC masiva deben ser diferenciadas la forma encefalítica de la no encefalítica, esta última tributaria de manejo antiparasitario, donde la neuroimagen muestra múltiples cisticercos viables pero con ausencia de edema, a diferencia de la forma encefalítica donde se evidencia un marcado edema y el uso de antiparasitarios podría solo exacerbar la reacción inflamatoria preexistente ${ }^{(19)}$. Por otro lado, el tiempo de tratamiento en estos casos tampoco es claro; García et al., demostraron que la efectividad de la terapia con albendazol es igual si se administra siete o 14 días, sin embargo, estos resultados pueden verse afectados dependiendo el número de quistes, como es el caso de las CCD ${ }^{(20)}$.

Concluimos que en este caso de CCD, al igual que en otros reportados, se decidió el manejo antiparasitario con albendazol y uso de corticoides, logrando una evolución clínica favorable y sin complicaciones durante la hospitalización.

Contribuciones de autoría: JMA y EC participaron en la concepción del artículo, JMA, EC y LC participaron en la redacción del artículo, JMA y LC participaron en la revisión crítica del artículo, todos los autores aprobaron el artículo para publicar.

Fuentes de financiamiento: autofinanciado.

Conflictos de interés: los autores declaran no tener conflictos de interés.

\section{REFERENCIAS BIBLIOGRÁFICAS}

1. García HH, Gonzalez AE, Evans CA, Gilman RH; Cysticercosis Working Group in Peru. Taenia solium cysticercosis. Lancet. 2003 Aug 16;362(9383):547-56.

2. García HH, Gonzales AE, Rodríguez $S$, Gonzalvez G, Llanos-Zavalaga F, Tsang VCW, et al. Epidemiologia y control de la cisticercosis en el Perú. Rev Peru Med Exp Salud Publica. 2010;27(4):592-7.

3. Saavedra H, Gonzales I, Alvarado MA, Porras MA, Vargas V, Cjuno RA, et al. Diagnóstico y manejo de la neurocisticercosis en el Perú. Rev Peru Med Exp Salud Publica. 2010;27(4):586-91.

4. Bhalla A, Sood A, Sachdev A, Varma V. Disseminated cysticercosis: a case report and review of the literature. J Med Case Rep. 2008 Apr 30;2:137. doi: 10.1186/1752-1947-2-137.

5. Khandpur S, Kothiwala SK, Basnet B, Nangia R, Venkatesh HA, Sharma R. Extensive disseminated cysticercosis. Indian J Dermatol Venereol Leprol. 2014 Mar-Apr;80(2):137-40. doi: 10.4103/0378-6323.129389.
6. Bern C, Garcia HH, Evans C, Gonzalez AE, Verastegui M, Tsang VC, et al. Magnitude of the disease burden from neurocysticercosis in a developing country. Clin Infect Dis. 1999 Nov;29(5):1203-9.

7. Kadhiravan T, Soneja M, Hari S, Sharma SK. Images in clinical tropical medicine: disseminated cysticercosis. Am J Trop Med Hyg. 2009 May;80(5):699

8. Park SY, Kong MH, Kim JH, Song KY. Disseminated cysticercosis. J Korean Neurosurg Soc. 2011 Mar;49(3):1903. doi: 10.3340/jkns.2011.49.3.190.

9. Foyaca-Sibat H, Ibanez-Valdes LdeF, Mashiyi MK. Disseminate cysticercosis. one-day treatment in a case. Electron J Biomed [Internet]. 2004 [citado el 15 de enero del 2014];3:39-43. Disponible en: http://biomed.uninet. edu/2004/n3/foyaca-n.html

10. Bastos AL, Marchiori E, Gasparetto EL, Andrade BH, Junior GC, Carvalho RC, et al. Pulmonary and cardiac cysticercosis: helical CT findings. $\mathrm{Br} \mathrm{J}$ Radiol. 2007 Mar;80(951):e58-60.
11. Garcia HH, Del Brutto OH. Heavy nonencephalitic cerebral cysticercosis in tapeworm carriers. The Cysticercosis Working Group in Perú. Neurology. 1999 Oct 22;53(7):1582-4.

12. Cavellani CL, Faleiros AC, Lino Rde $S$ Jr, Dos Reis MA, Teixeira Vde P. Cysticercosis in the elderly. Ann Diagn Pathol. 2007 Oct;11(5):330-3.

13. Sander HW, Castro C. Images in clinical medicine. Neurocysticercosis. N Engl J Med. 2004 Jan $15 ; 350(3): 266$.

14. Bustos JA, Garcia HH, Dorregaray R, Naranjo M, Pretell EJ, Gonzalez AE, et al. Detection of muscle calcifications by thigh CT scan in neurocysticercosis patients. Trans R Soc Trop Med Hyg. 2005 Oct;99(10):775-9.

15. Wallin MT, Pretell EJ, Bustos JA, Caballero M, Alfaro M, Kane R, et al. Cognitive changes and quality of life in neurocysticercosis: a longitudinal study. PLoS Negl Trop Dis. Jan;6(1):e1493. doi: 10.1371/ journal.pntd.0001493. 
16. Garcia HH, Del Brutto OH; Cysticercosis Working Group in Peru.. Neurocysticercosis: updated concepts about an old disease. Lancet Neurol. 2005 Oct;4(10):653-61.

17. Singh N, Singh DK, Parihar A, Singh R. Disseminated cysticercosis: rare manifestation of a common disease. BMJ Case Rep. 2012 Dec 18;2012. doi: 10.1136/bcr-2012-007876.

18. Budke CM, White AC Jr, Garcia HH. Zoonotic larval cestode infections: neglected, neglected tropical diseases? PLoS Negl Trop Dis. 2009;3(2):e319. doi: 10.1371/journal.pntd.0000319.

19. Del Brutto OH, Campos X. Massive neurocysticercosis: encephalitic versus non-encephalitic. Am J Trop Med Hyg. 2012 Sep;87(3):381. doi: 10.4269/ ajtmh.2012.12-0162.

20. Garcia HH, Gilman RH, Horton J, Martinez M, Herrera G, Altamirano $\mathrm{J}$, et al. Albendazole therapy for neurocysticercosis: a prospective double- blind trial comparing 7 versus 14 days of treatment. Cysticercosis Working Group in Peru. Neurology. 1997 May;48(5):1421-7.

Correspondencia: Julio Maquera Afaray Dirección: Unidad de infectologia, Hospital Nacional Guillermo Almenara Irigoyen. Av. Grau N. ${ }^{\circ} 800$, Lima 13, Perú.

Teléfono: 51-1 324-2983 anexo 44082

Correoelectrónico:vigo_ma@hotmail.com

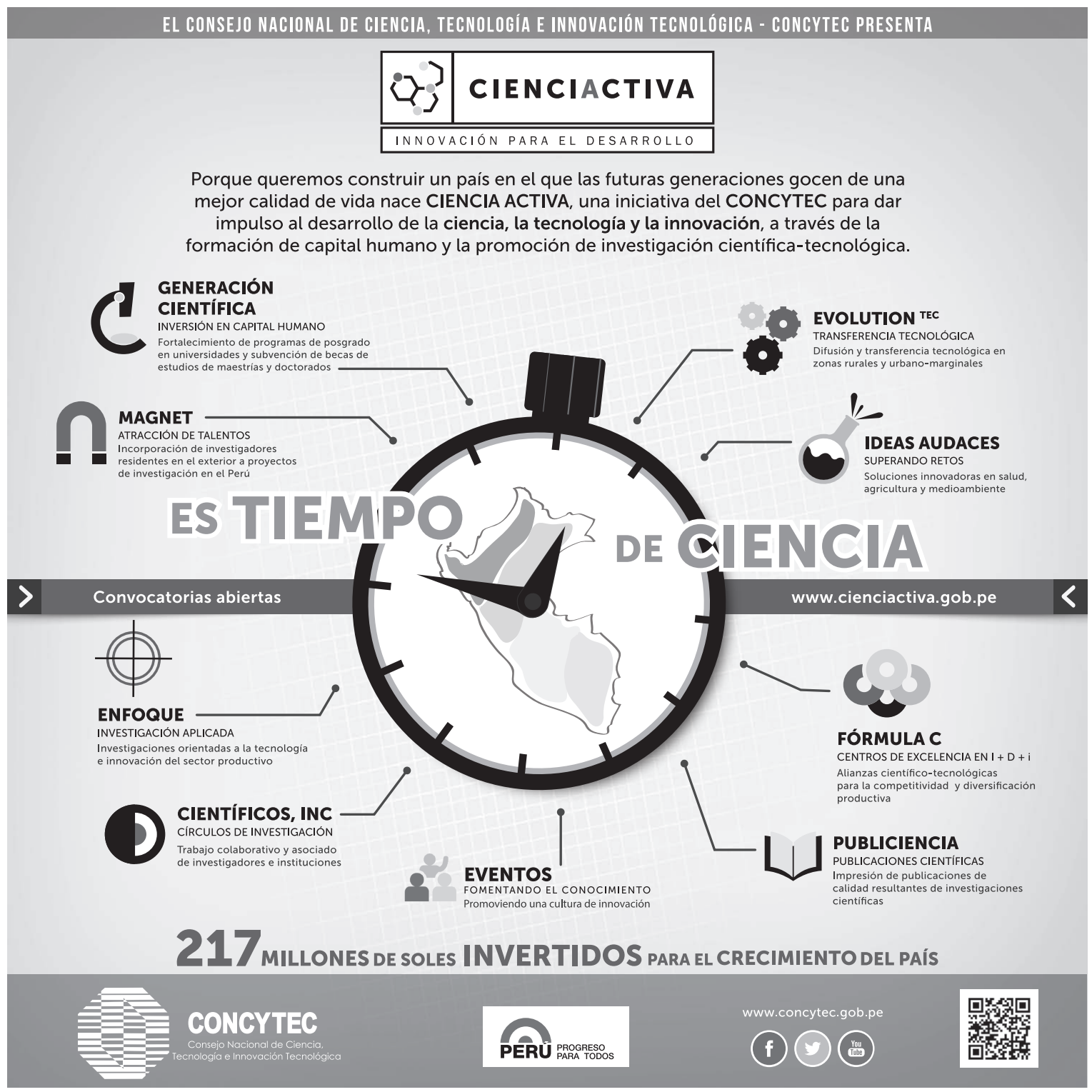

